\title{
Quality Aware Reliable Messaging For Wireless Mesh Network
}

\author{
Ms. Anuradha Desai ${ }^{1}$, Dr. Santosh Deshpande ${ }^{2}$ \\ ${ }^{1}$ (Department of Computer Network Engineering, Vishveshwarya technological University, Belgaum, India) \\ ${ }^{2}$ (Department of Computer Network Engineering, Vishveshwarya technological University, Belgaum, India)
}

\begin{abstract}
Wireless mesh network is an emerging technology progressing in the field of wireless networking. The broadcast nature of wireless mesh network allows each router to search destination and forward the packet. Wireless mesh network is based on IEEE 802.11 standard according to which the received packet must be acknowledged and the routers unacknowledged continue broadcasting packet in their range. This leads to signaling overhead and decreases throughput of the network. The proposed work overcomes this signaling overhead in addition to the design of a routing metric, expected forwarded counter [EFW] which deals with the problem of selfish behavior of mesh routers in wireless mesh network. EFW considers forwarding behavior of node and wireless link quality to select the most reliable and high performance path. The proposed system is evaluated by performing comparative analysis with the existing system by considering performance metrics like packet delivery ratio, average throughput, bandwidth utilization. The proposed work shows significant increase in throughput with efficient bandwidth utilization and the metric EFW selects a path with highest delivery rate, considering both quality of wireless links and reliability of network node in wireless mesh network.
\end{abstract}

Keywords: Data dropping, metrics, broadcasting, wireless mesh network.

\section{Introduction}

Wireless Mesh Network (WMN) is an emerging technology making progress in the field of wireless networking. Wireless mesh network architecture consist of mesh routers (MRs), mesh clients (MCs) and multiple internet gateways (IGWs). Mesh routers act as a bridge to forward packet until it reaches destination. Mesh routers have minimal mobility and are linked to one another to extend the network coverage. The clients could be either stationary or mobile. If mesh client wants to communicate with another node that is not within its communication range, it forwards the packet to its nearest mesh router. The packet is then forwarded over a mesh router backbone according to a routing protocol, until it reaches the router that can forward packet to the destination node.

Wireless mesh network and ad hoc network have some features in common, thus the routing protocols developed for ad hoc network can be applied for Wireless mesh network. But these routing protocols must include some performance metrics, since the power requirements and mobility features of wireless mesh networks are different from ad hoc networks [1]

The broadcast nature of wireless mesh network allows each router to search destination and forward the packet. Wireless mesh network is based on IEEE 802.11 standard according to which the received packet must be acknowledged and hence, when the destination node receives a packet, it acknowledges the respective router. The router can hear acknowledge signals received by its neighboring routers. The routers which do not receive acknowledgement continue to broadcast packet in its range. This creates signaling overhead and decreases throughput of the network [8].

Expected transmission count [ETX] is a routing metric that represents the transmission reliability of a wireless link. ETX measures the expected number of transmissions and retransmissions needed to send a unicast packet correctly over a wireless link. The successful transmission of a packet is referred to the transmission of the packet in one direction and reception of an acknowledgement in the reverse direction [3], [4]. ETX is determined by active probing, in which the number of packets received successfully are compared with the number of packets sent in a given time [2]. This metric is designed assuming that each mesh router participates honestly in the forwarding process. In wireless mesh network, a selfish node that provides connectivity through its router might try to greedily consume the available bandwidth by dropping packets sent by other nodes. In presence of such routers, ETX metric fail to select the network path with highest delivery rate [3].

The proposed work focuses on, first to overcome the signaling overhead of the routers in their range that does not receive acknowledgement. Second, design of the metrics that selects highest delivery rate considering both the quality of wireless links and reliability of network node.

\section{System Design}

The broadcast nature of wireless mesh network enables gateway to forward a packet to its nearest routers in the network. When an intermediate router receives a packet, it broadcasts the packet to other nodes 
within its range and to the neighboring routers. The routers that receive the first packet add it to their queue. The router increments its forward counter by 1 after forwarding the packet. When the packet reaches destination, it must reply with an acknowledgement to notify successful reception, according to IEEE 802.11 standard. The packet forwarded path is used to reply acknowledgement packet and then increments its acknowledgment counter by 1 . The routers which do not receive acknowledgement continue to broadcast packet in its range. This creates signaling overhead and decreases throughput of the network. To avoid the unnecessary signaling of routers, the number of routers unacknowledged is counted and with the help of timer and acknowledgment counter the unnecessary signaling can be stopped. This leads to an increase in overall throughput of the network.

Consider an example with respect to Fig 1. A packet is forwarded from gateway to the nearest mesh routers (MR1, MR6). These routers forward the same packet to their neighboring routers (MR3, MR4, MR5, and MR7) and at the same time, they broadcast the packet to the nodes within their range. Each router that forwards the packet updates its forward counter. Consider the destination node is in the range of MR5, the packet from gateway traverses the routers MR1, MR3, MR5 and finally reaches destination. These router increments their forward counter by 1 . After receiving packet, the destination node replies back to MR5 with an acknowledgement traversing the same path in reverse direction. After receiving this acknowledgement, the router MR5 increments their acknowledgements counter by 1 . The same acknowledgement packet is forwarded by MR5 to MR3 and as a result MR3 also increments their acknowledgements counter by 1 . Similarly, the acknowledgement packet is received by MR1, counter is updated and then acknowledgement reaches the gateway.

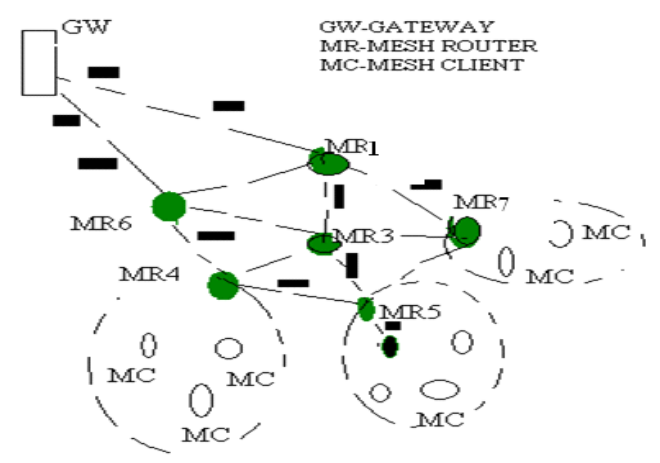

Fig 1 Forwarding packet in the network

The routers (MR7, MR4 and MR6) which are not in the acknowledgement path continue broadcasting the packet within their range. This leads to signaling overhead and degradation of network performance. To overcome this, a timeout can be set to stop signaling with respect to acknowledgement counter.

The forward counter is denoted by Cij (fwd)

The acknowledgement counter is denoted by $C i j$ (ack)

[Number of nodes did not received acknowledgement $]=[$ Number of nodes forwarding packet]-[number of nodes acknowledged]

$C=\left[\sum c x(f w d)-\sum c x(a c k)\right]$ ( $x=0$ to $n$ number of nodes)

If acknowledgment counter is zero and timeout exceeds the threshold value, the packet is de-queued and the router stops signaling.

If Cack $=0$ \&\& timer to receive ack $>$ threshold value, then dequeue the packet

Routing Metrics

The routing metrics have been designed to select the most reliable path in wireless mesh network. These metrics do not consider the presence of a selfish router that drops the packets sent by other nodes. Routing metric ETX adopt a probabilistic model to represent the transmission and retransmission. To calculate ETX, it is necessary to estimate packet loss probability in both directions, since wireless mesh network is based on IEEE 802.11 and the destination must acknowledge each data frame received. Let $(\mathrm{i}, \mathrm{j})$ be a wireless link between nodes $\mathrm{i}$ and $\mathrm{j}, p i j$ and $p j i$ denote the packet loss probability of wireless link (i,j) in forward and reverse direction respectively [3]. Thus the probability of successful transmission on wireless link $(\mathrm{i}, \mathrm{j})$ can be computed as

$P s, i j=(1-p i j)(1-p j i)$

$p i j$ is forward packet loss probability

$p j i$ is reverse packet loss probability

Probability of successful transmission is $p s, i j=(1-p i j)(1-p j i)$ 
The expected number of transmissions required to deliver the data packet, considering both transmission and successive acknowledgement can be evaluated as,

$E T X=1 / P s, i j=[(1-p i j)(1-p j i)]$

ETX selects the most reliable path but does not model accurate delivery rate of the network link, as the forwarding behavior of nodes that have established the link is not considered. In particular, ETX and its derived metrics do not take into account that a selfish node might discard the packet after its correct reception, if the selfish node benefits from not forwarding it.

A selfish node may drop packets sent by other nodes at network layer instead of data link layer, after the reception of data frame and successive transmission of acknowledgement. If the selfish node does not send acknowledgement after reception of data frame, the sending node will increase the packet loss probability in reverse direction, $(p r, i j)$ and this selfish action will be considered in ETX metric by lowering data-link layer reliability. To overcome the problem caused by dropping behavior of selfish participants, combine the link quality measured by ETX routing metrics with the forwarding reliability of relaying node $\mathrm{j}$. Let $(p r, i j)$ be the dropping probability of network node $\mathrm{j}$ at network layer, then $p f, i j=(1-p d, i j)$ represents its forwarding probability. A network node can drop selectively the traffic sent by its neighboring nodes. The dropping probability of node $\mathrm{j}$ is identified by both sending node $\mathrm{i}$ and forwarding node $\mathrm{j}$. The probability that a packet sent through a node $\mathrm{j}$ is successfully forwarded can be computed as: $p f w d, i j=(p s, i j) .(1-p d, i j)$.

The number of transmissions necessary to have the packet successfully forwarded (Expected Forwarded Counter) EFW can be measured as:

$E F W=1 /(p f w d i j)=[1 /[(1-p i j)(1-p j i)]] .[1 /(1-p d, i j)]$

First part of equation provides quality of physical and MAC layer. Network layer reliability is assured by the second part. EFW represents both physical condition of wireless medium and selfishness of nodes with which link is established [3].

\section{Result Analysis}

Packet Delivery Ratio: Packet delivery ratio is defined as the ratio of successfully delivered data packets. Packet delivery ratio $=(($ send packets - dropped packets $)) /$ Send packets

Throughput: Throughput is the total data packets received by the receiver at a particular Unit Time. Throughput $=$ bytes $($ received packets $) * 8 /$ (Finish time - Start time $)$
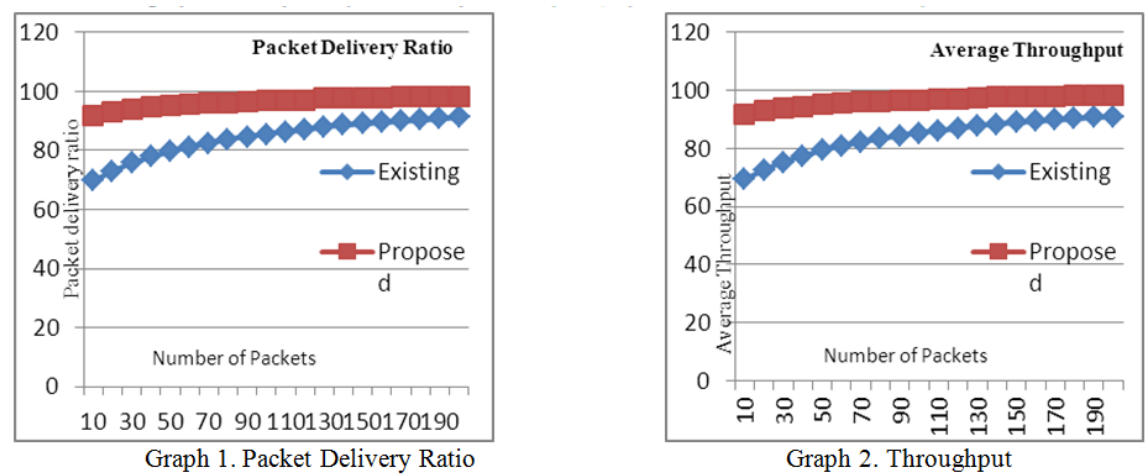

The graphs show that the packet delivery ratio, throughput and bandwidth utilization is higher for proposed system than the existing system. This indicates that the number of packets dropped is reduced and as a result there is increase in throughput. When the packets are delivered successfully without dropping, the number of retransmission of packet reduces which also reduces the time required for successful transmission of packets. As a result, more number of packets are delivered successfully in short period and effective utilization of bandwidth is achieved.

Bandwidth utilization: bandwidth utilization is defined as the efficient use of available bandwidth to achieve specific goal.

Bandwidth utilization $=100-($ dpackets - data packets $) /$ dpackets $* 100$

Time: Time required for successful transmission of packets in seconds

Time $=$ Transfer time $/$ sequence number $* 100$ seconds 


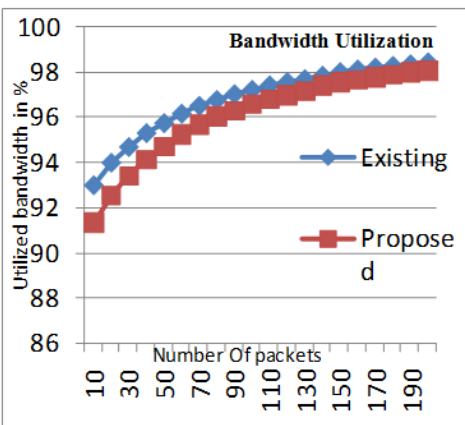

Graph 3. Bandwidth Utilization

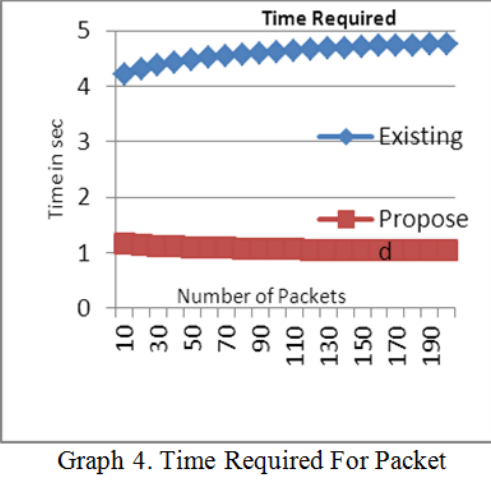

Graph 4. Time Required For Packet

Packet dropped: Dropped packet: The number of packets dropped due to congestion in the network.

$$
\text { Dropped packets }=\sum \text { The Number of dropped packets }
$$

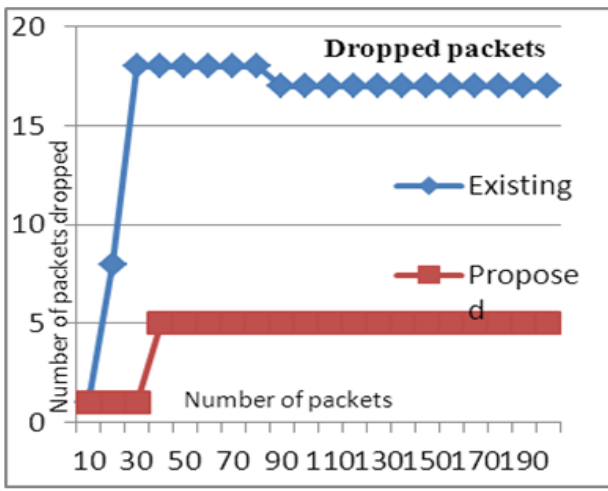

Graph 5. Packet Dropped

Packet Delivery Ratio [EFW]: The ratio of the number of delivered data packets to the destination $P D R=\sum$ number of data packets receive $/ \sum$ number of data packets send

Average Throughput[EFW]: Total number of packets received in a particular time.

Throughput $=$ bytes $($ received packets $) * 8 /($ Finish time - Start time $)$

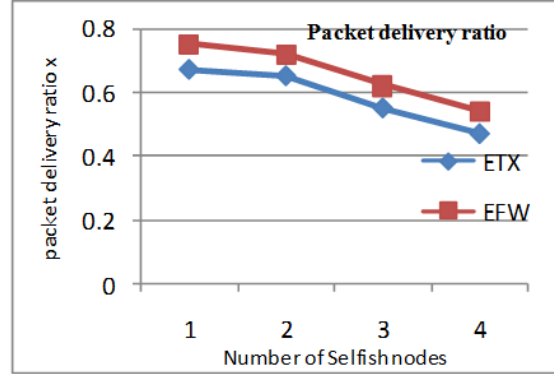

Graph 6. Packet Delivery Ratio

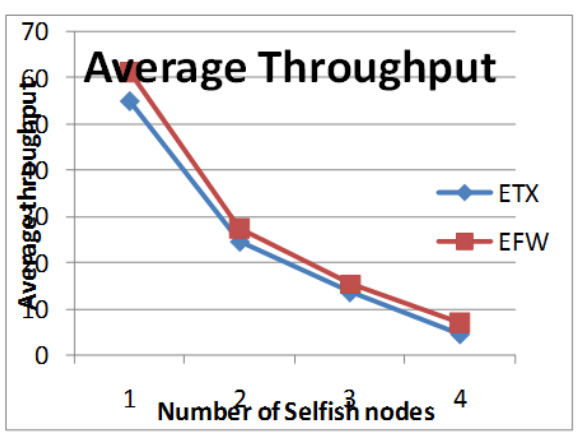

Graph 7. Average Throughput for ETX and EFW

\section{Conclusion}

The broadcast nature of IEEE 802.11 based wireless mesh network allows each router to search destination, forward the packet and wait for acknowledgement. The routers which do not come in acknowledgement path continue signaling in its range. This creates signaling overhead which degrades the overall performance of the network. To overcome this, the proposed system counts the number of routers unacknowledged and with the help of acknowledgement counter and timer, the amount of signaling overhead is reduce. The simulation result shows: 1) Increase in packet delivery ratio by $18 \%$ and improve in average throughput by $18 \%$. 2) Bandwidth utilization is $2 \%$ less than existing system which results in proper channel usage 3) Time required for packet transmission is 3 seconds less than existing system and 4) packet dropped is constant but less for proposed system compared to existing system. Thus the proposed system has better network performance than the existing system.

The ETX metric fails to select the network path with highest delivery rate in presence of selfish nodes. 
To overcome this, the routing metrics EFW and its two variants, MEFW and JEFW are proposed to select a path considering both quality of wireless links and forwarding behavior of network nodes. The result shows that the proposed metric EFW increases throughput and packet delivery ratio when compared to ETX metric.

\section{References}

[1] I.F Akyildiz and Wang, A Survey on Wireless Mesh Networks (IEEE commun.Mag vol. 43, no.9, pp.s23-s30, sep.2005)

[2] Sakhapure S.S.,Channe H, A Cross Layer Routing Approach In Self Configurable Wireless Mesh Networks, International journal of engineering research and technology (IJRET) vol.1 Issues 8,October-2012 ISSN:2278 -0181.

[3] Stefano Paris,Cristina Nita -Rotaru, Fabio Martignon and Antonio Capone, Cross Layer Metrics For Reliable Routing In Wireless Mesh Network.

[4] MdAsri Bin NgadiSaquib Ali Abdul Hanan Abdullah and Rashid Hafeez Khokhar, A Taxanomy Of Cross Layer Routing Metrics for Wireless Mesh Network.

[5] Jaydip Sen. and Kaustav Goswami, An Algorithm for Detection of Selfish Nodes in Wireless Mesh Network,Innovations labs TCS.

[6] Xiaolin Cheng, Prasant Mohapatra, Retransmission-Aware Queuing and Routing For Video Streaming In Wireless Mesh Networks.

[7] Vighneshwar Rao Gannapathy Ahamed Fayeez Bin Tuani Ibrahim Zahirlahda Bin Zakaria, An Enhancement of RTS/CTS Control Handshake in CSMA/CA Based MAC Protocol for an Efficient Delivery over Multihop Network, International Journal of Research in Engineering and Technology ISSN 2319-1163/pISSN $2321-7308$.

[8] Wireless Mesh Networks: WMN Overview, WMN Architecture, International Conference on Communication Engineering and Networks IPCSIT Vol.19 2011, IACSIT Press, Singapore.

[9] Stefano Paris Cistina Nita Rotaru Senior Member IEEE, EFW Cross Layer Metrics for Reliable Routing In Wireless Mesh Network With Selfish Participants. 